\title{
Accuracy of Digital Vs Conventional Implant Impressions for an All-on-Four Treatment Concept
}

\author{
Antonio Afram ${ }^{1}$, Anne Christelle Makhlouf ${ }^{1}$, Paul Boulos ${ }^{2}$ and Elie \\ Nasr $^{1 *}$ \\ ${ }^{1}$ Department of Fixed Prosthesis and Occlusodontics of the Faculty of Dentistry at \\ Saint Joseph University, Beirut, Lebanon \\ ${ }^{2}$ Department of Removable Prosthesis of the Faculty of Dentistry at Saint Joseph \\ University, Beirut, Lebanon \\ *Corresponding Author: Elie Nasr, Department of Fixed Prosthesis and \\ Occlusodontics of the Faculty of Dentistry at Saint Joseph University, Beirut, \\ Lebanon.
}

Received: September 27, 2021

Published: October 28, 2021

(C) All rights are reserved by Elie Nasr., et al.

\section{Abstract}

Aim: The aim of this article is to compare two impression techniques: digital and conventional, in the case of an All-on-Four implantsupported hybrid prosthesis, in order to draw a clinical conclusion regarding the accuracy of adaptation of the prosthetic reconstructions.

Materials and Methods: On a prototype maxillary model made of an extra hard white acrylic resin, were placed four Straumann ${ }^{\circledR}$ Bone Level implants with the Regular CrossFit ${ }^{\circledR}$ connection (RC): as a $4.1 \mathrm{~mm}$ in diameter by $10 \mathrm{~mm}$ of length. Based on the Allon-Four concept, the two implants at the lateral incisors $(12,22)$ were placed in a straight axis, while the other two implants were inclined 45-degrees distally at the level of the second premolars $(15,25)$. Thus, on this prototype model, twenty impressions were made including ten impressions taken using the Trios ${ }^{\circledR} 3$ (3Shape), and ten conventional impressions were obtained using a custommade open-tray and the Impregum Penta ${ }^{\circledR}$ (3M-ESPE) as a materiel of choice. Subsequently, digitalization of plaster models obtained from conventional impressions was required, to allow the superimposition of digital and conventional impressions with the control model so as to compare the accuracy of the two different impressions techniques.

Results: This study showed that horizontal and vertical mean inaccuracies were significantly different at the anterior and posterior sites for digital and conventional impressions (-p-value $<0.05)$, indicating that digital impressions deviations were smaller than those associated with conventional techniques.

Conclusion: For a model with four implants following the All-on-Four concept, the digital impression proves to be more precise and more reliable than the conventional impression.

Clinical Significance: For an All-on-Four treatment concept, digital impression techniques are recommended, demonstrating clinically acceptable outcomes.

Keywords: All-on-Four; Implants; Precision; Reliability; Digital Impression; Intraoral Scan; Conventional Impression; Polyether 


\section{Introduction}

Today, in the context of a fixed prosthesis, the All-on-Four concept; prosthesis screwed onto four implants, developed by Paulo Malo in the early 1990s, is experiencing a well-deserved boom $[1,2]$. Indeed, it turns out to be an efficient and cost-effective treatment for the esthetic and functional rehabilitation of a completely edentulous arch [3]. However, a good impression is essential to obtain a precise master model, which is the key to the success of this future implant-supported prosthesis.

The digital revolution, which has only just begun, plunges the practitioner into a digital universe, which is becoming more and more imposing. This powerful and sophisticated evolution offers very significant productivity gains in the field of dentistry. Nowadays, advances in the IT field make it possible to digitally manage the production processes for this type of dental prosthesis, particularly with Computer Aided Design and Manufacturing (CAD/CAM), by offering an acceptable clinical adaptation or even better comparing it to the conventional impressions and fabrication methods [4].

The impression is in fact the main vector of information between the practitioner and the prosthesis laboratory. Whether conventional or digital, impressions must meet specific requirements and obey many parameters. Hence, a comparative synthesis of the two methods is necessary before considering the use of the intraoral scanner as a reliable alternative to conventional impressions. However, we must not forget that progress is founded on tradition and that conventional techniques are the basis for everything we hold today.

\section{Aim of the Study}

Thus, the aim of this article is to compare two impression techniques: digital and conventional, in the case of an implant-supported hybrid prosthesis of the All-on-Four type, in order to draw a clinical conclusion concerning the precision of adaptation of prosthetic reconstructions.

\section{Materials and Methods \\ Prototype model}

An initial prototype model designed with an extra hard white acrylic resin, was a representation of an upper edentulous arch on which four Straumann ${ }^{\circledR}$ Bone Level implants with the Regular CrossFit $^{\circledR}$ connection (RC): as a $4.1 \mathrm{~mm}$ in diameter by $10 \mathrm{~mm}$ of length, were implanted in a straight axis (Figure $1 \mathrm{~A}$ and $1 \mathrm{~B}$ ).
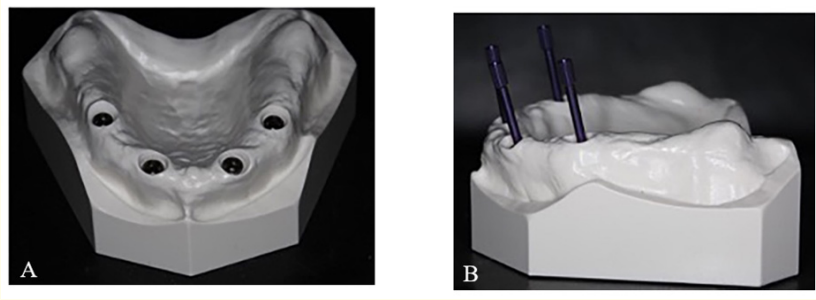

Figure 1: Initial prototype model comprising four straight implants. A: Occlusal view; B: Profile view.

In order to simulate an All-on-Four treatment concept [5] the two distal implants will have to be explanted using a surgical trephine with a diameter of $10 \mathrm{~mm}$ and repositioned distally along an axis of 45 degrees using a surveyor and an acrylic resin type Formatray from $\operatorname{Kerr}^{\circledR}$ (Figure 2A and 2B).
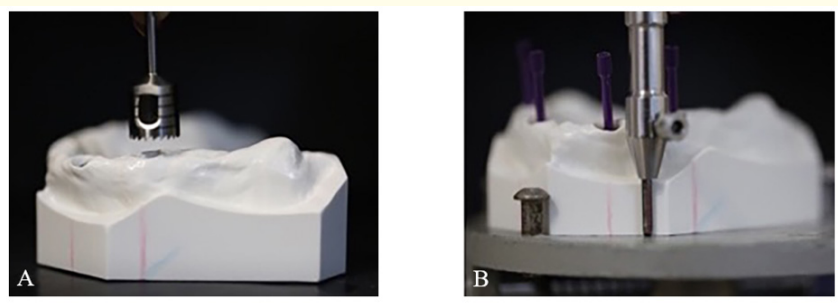

Figure 2: Initial prototype model under modification. A: Explanation of the distal implant; B: Repositioning of the implant using a surveyor.

This will then lead to the final prototype model which was comprised of two straight implants at the level of the lateral incisors $(12,22)$ and two others inclined 45 degrees distally at the level of the second premolars $(15,25)$ (Figure $3 \mathrm{~A}$ and $3 \mathrm{~B}$ ).

On the final prototype model obtained, in order to facilitate the subsequent superposition of the digitized impressions, three metal 

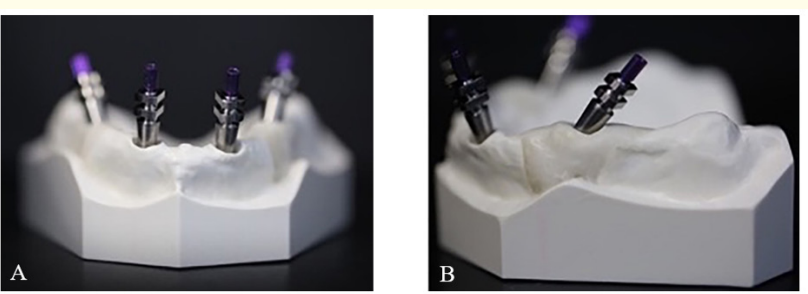

Figure 3: Final prototype model with two straight implants and two more angled 45 degrees distally. A: Front view; B: Profile view.

balls with a $2 \mathrm{~mm}$ diameters, measured using a thickness gauge, were fixed in the center of the dental crest on the master model between the four implants, equidistant from each two adjacent implants. In other words, they were placed on the perpendicular bisector, of the line joining the facing proximal ends of the juxtaposed implants by passing through their respective centers. Thus, these three small-caliber metal balls will serve as fixed references for the superposition of the subsequently digitized models [6,7] (Figure 4).

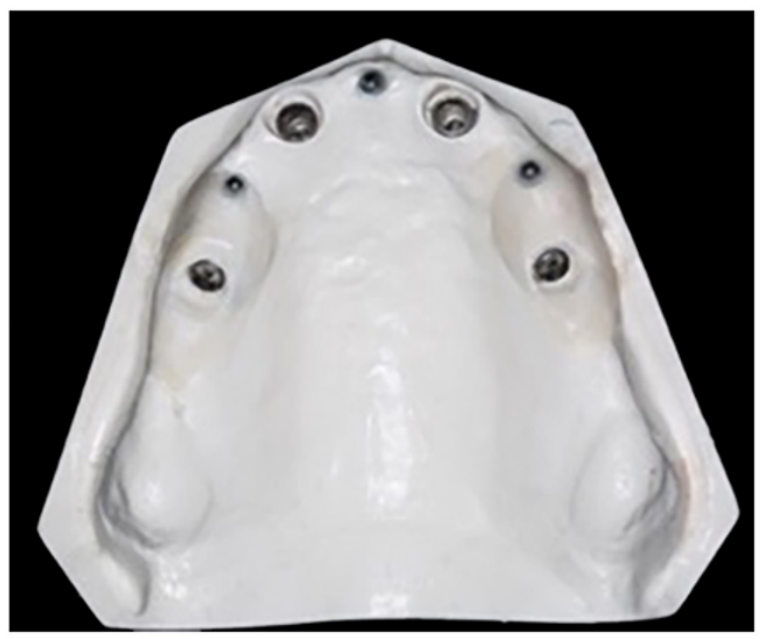

Figure 4: The three metal balls in place.

\section{Control model}

After fixing the metal balls, the prototype model was scanned ten times using an E3 desktop scanner (3Shape) [8], following the installation and tightening at $10 \mathrm{Ncm}$ of the appropriate $\mathrm{RC}$ Straumann ${ }^{\circledR}$ CARES $^{\circledR}$ Mono Scanbodies. Before each scan and in order to minimize the margins of error, the scanner was calibrated according to the manufacturer's recommendations [9].

After obtaining the ten scans, the deviation value also known as the difference between the various acquired virtual models, was measured by their superimpositions using a specific program: the 3Shape Dental System ${ }^{\circledR}$. Zero values between the various models ranging from T1 to T10 were recorded. In what will follow, the choice of the control model will be made randomly. The T8 was chosen to be the control model of the study.

\section{Conventional impression of the prototype model}

Using a perforated metallic stock tray, a primary impression of the prototype model was taken with alginoplast ${ }^{\circledR}$ (Heraeus Kuzler) for the realization of an open custom-made impression tray. The latter was be more precise spaced from the plaster model obtained by placing two layers of wax on the palate and four supracrestal layers in the form of a U-shaped rim. In order to gain stability, three stops including two tuberosities and one palatal were put in place [10].

The engaging RC open-tray impression copings, by the shape of their internal hexagon, have been modified using a transmetal bur, into non-engaging transfer copings in order to facilitate the disinsertion of the impression, by reducing friction at the implanttransfer interface [11].

Group 1: Once the four non-engaging copings are accurately placed into their respective implant position on the prototype model and torqued at $15 \mathrm{Ncm}$, the custom-made open-tray was adjusted and properly adapted and the inner surface of the tray was adhesive painted for better impression material retention, the impression was taken with a Polyvinyl Siloxane material (Express Putty and Light (3M-ESPE)). Its casting will make it possible to obtain a replica of the prototype model, from which ten custom-made open-tray similar to their antecedent will be obtained, allowing the minimization of the error biases linked to an excessive amount of impression material [12].

Citation: Elie Nasr., et al. "Accuracy of Digital Vs Conventional Implant Impressions for an All-on-Four Treatment Concept". Acta Scientific Dental Sciences 5.11 (2021): 98-106. 
Group 2: The non-engaging transfer copings were placed and torqued at $15 \mathrm{Ncm}$ on the plaster model from the impression taken earlier with the Polyvinyl Siloxane: Express Putty and Light (3MESPE). They were then connected using a stainless-steel wire with a diameter of $0.6 \mathrm{~mm}$ as well as a low shrinkage acrylic resin (Pattern Resin LS from GC America Alsip, IL, USA).

After a week, the resin was cut and welded again using the same product but this time, after placing and torquing the non-engaging transfer copings at $15 \mathrm{Ncm}$ on the prototype model, in order to simulate the clinical situation as much as possible. In this way the acrylic distortions were reduced to minimum [13]. Once the fit of the custom-made open-tray impression was verified, the impression was ready to be taken with Impregum Penta ${ }^{\circledR}$ (3M-ESPE). Two layers of adhesive were coated on the intaglio surface of the trays, while the prototype model was lubricated using an isolating medium $[14,15]$. The Impregum Penta ${ }^{\circledR}$ (3M-ESPE) was first injected around the transfer copings using a specific syringe, before seating the impression tray loaded with the same polyether product. In this way, ten conventional impressions were made of the prototype model, noting that each transfer was for single use in order to reduce the risk of error.

From these polyether impressions, ten plaster models (CAN Stone N-Siladent-Germany) were obtained, prepared under vacuum according to the manufacturer's instructions and cast on a vibrator in order to reduce the risks of air bubbles forming as well as other errors. Subsequently, each model was scanned using an E3 desktop scanner (3Shape), after seating and tightening at $10 \mathrm{Ncm}$ the proper RC Straumann ${ }^{\circledR}$ CARES $^{\circledR}$ Mono Scanbodies. Thus, from the ten plaster models resulting from conventional impressions, ten virtual models emerged, i.e. P1 - P10.

\section{Digital impression of the prototype model}

On the prototype resin model, the RC Straumann ${ }^{\circledR}$ CARES ${ }^{\circledR}$ Mono Scanbodies were positioned on a way that their bevels were buccally oriented, they were then torqued at $10 \mathrm{Ncm}$. Using the Trios ${ }^{\circledR}$ 3 (3Shape), ten digital impressions were taken by a single operator with standardized scanning technique, after the calibration of the device before each scan in order to reduce error biases [16]. Ten digital models have then immediately emerged, i.e. D1 - D10.
Superposition of the conventional and digital impressions with the control model

Using a specific program: the 3Shape Dental System ${ }^{\circledR}$, the superposition of the balls from the various impressions led to the comparison of the virtual models [17]. Thus, the three balls on the control model: T8, were colored red, green and blue, from left to right successively.

Starting with the conventional impressions; on the P1 model the three balls which correspond to the three others already marked on the control model: T8, were also colored in red, green and blue respectively.

Finally, the realization of the superposition was completed by aligning the red balls then the green and at the end the blue ones in order to obtain a single model representing P1 and T8 superimposed (Figure 5).

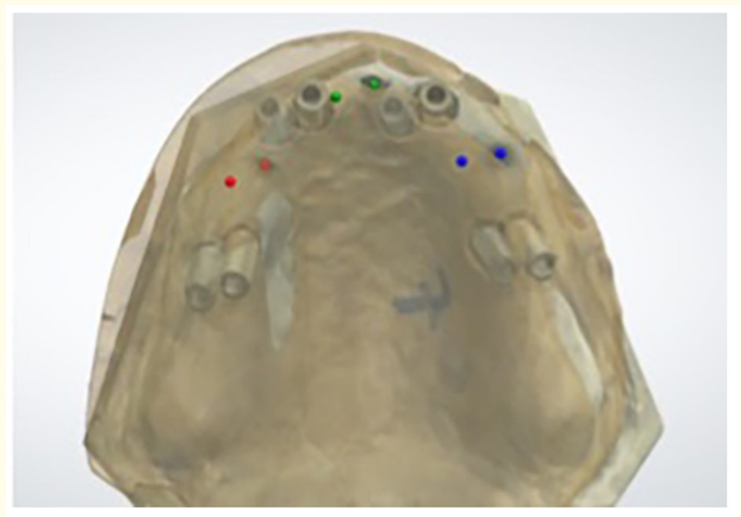

Figure 5: Superposition of P1 and T8.

In order to standardize the measurements so as not to have biased errors, the cuts on the superimposed models were made while remaining on a magnification of $250 \%$. The vertical and horizontal cuts required the use of a pre-measured grid.

The vertical cuts were made just in the middle of the transfer copings. At their most vestibular plane and $4 \mathrm{~mm}$ from the implant neck, the measurement was evaluated (Figure 6A). 
As for the horizontal ones, they were made tangentially to the transfer copings. At the level of their most mesial point and $4 \mathrm{~mm}$ from the implant neck, the measurement was assessed (Figure 6B).
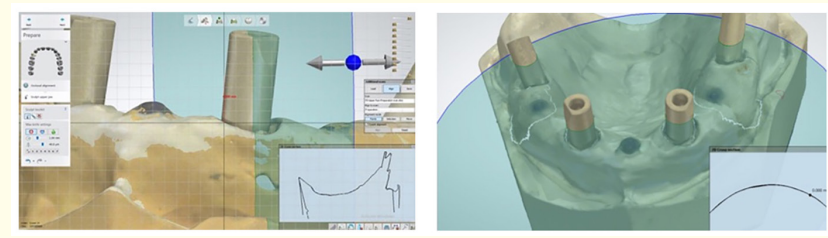

Figure 6: Measurement cuts. A: Vertical; B: Horizontal.

Note that the standards applied to conventional impressions were themselves adapted to the digital impressions. Subsequently, the values obtained represented the difference between the position of each implant resulting from one of the two impression techniques and the control model.

\section{Statistical analysis}

SPSS for Windows statistical software (Chicago, IL, USA, version 25.0) was used for statistical analysis of the data (Table 1). The significance level adopted corresponds to -p-value $\leq 0.05$.

\begin{tabular}{|l|c|}
\hline Control Model T8 \\
\hline $\begin{array}{l}\text { Conventional Impressions } \\
\text { Digitized }\end{array}$ & $\begin{array}{c}\text { Digital } \\
\text { Impressions }\end{array}$ \\
\hline P1 & D1 \\
\hline P2 & D2 \\
\hline P3 & D3 \\
\hline P4 & D4 \\
\hline P5 & D5 \\
\hline P6 & D6 \\
\hline P7 & D7 \\
\hline P8 & D8 \\
\hline P9 & D9 \\
\hline P10 & D10 \\
\hline
\end{tabular}

Table 1: Comparison protocol.
First, the Kolmogorov-Smirnov tests were performed in order to assess the normality of the distribution of quantitative variables. Subsequently, the parametric tests were performed for the variables which follow the normal distribution. Next, the non-parametric tests were carried out for the variables which do not follow the normal distribution. Student's and Mann-Whitney tests were performed to compare the inaccuracies between the two impression techniques. Finally, the One-Sample t tests were used to compare the mean values of imprecision with the theoretical value 0 which assumes maximum precision.

\section{Results}

The mean, the standard deviation, the minimum and maximum of imprecision of the various measurements carried out at the level of the models resulting from the two impression techniques in the horizontal and vertical directions were described in the following table (Table 2).

In the vertical direction (V), the mean was significantly lower for models acquired from digital impressions compared to the models issued from the conventional impressions, and this at the level of anterior sites $12(-p$-value $=0.011), 22(-p$-value $=0.022)$ and posterior ones 15 (-p-value <0.001), 25 (-p-value = 0.047).

In the horizontal direction $(\mathrm{H})$, the mean was significantly lower for models from digital impressions compared to the models obtained through conventional impressions, and this is at the level of the anterior site $22(-p$-value $=0.048)$ and posterior ones $15(-p$ value $=0.05), 25(-p$-value $=0.024)$. Note that for the anterior site 12 , although the imprecision was lower in the models issued from digital impressions, the difference was not so significant (-p-value $=0.089$ ).

This study showed that the mean vertical and horizontal inaccuracies were significantly different from the theoretical value 0 (which assumes maximum precision), at the level of the anterior $(12,22)$ and posterior $(15,25)$ sites for the models acquired from both impression techniques (-p-value < 0.05) (Table 3).

\section{Discussion}

Nowadays, the technological advances in the dental field push us to wonder about the reliability or even the precision of the new tools put on the market [18]. Indeed, the in vitro data generated 


\begin{tabular}{|c|c|c|c|c|c|c|c|}
\hline Zones & & $\mathbf{N}$ & Mean & $\begin{array}{l}\text { Standard } \\
\text { Deviation }\end{array}$ & Minimum & Maximum & p-value \\
\hline \multirow{2}{*}{$12 \mathrm{~V}$} & Digital & 10 & .036 & .0160 & .010 & .052 & \multirow{2}{*}{.011} \\
\hline & Conventional & 10 & .079 & .0454 & .031 & .184 & \\
\hline \multirow{2}{*}{$15 \mathrm{~V}$} & Digital & 10 & .029 & .0204 & .006 & .067 & \multirow{2}{*}{.000} \\
\hline & Conventional & 10 & .106 & .0502 & .020 & .160 & \\
\hline \multirow{2}{*}{$22 \mathrm{~V}$} & Digital & 10 & .028 & .0157 & .010 & .059 & \multirow{2}{*}{.022} \\
\hline & Conventional & 10 & .100 & .0897 & .006 & .291 & \\
\hline \multirow{2}{*}{$25 \mathrm{~V}$} & Digital & 10 & .052 & .0288 & .014 & .100 & \multirow{2}{*}{.047} \\
\hline & Conventional & 10 & .092 & .0513 & .037 & .200 & \\
\hline \multirow{2}{*}{$12 \mathrm{H}$} & Digital & 10 & .051 & .0299 & .010 & .120 & \multirow{2}{*}{.089} \\
\hline & Conventional & 10 & .085 & .0555 & .008 & .196 & \\
\hline \multirow{2}{*}{$15 \mathrm{H}$} & Digital & 10 & .027 & .0231 & .007 & .079 & \multirow{2}{*}{.005} \\
\hline & Conventional & 10 & .105 & .0740 & .013 & .283 & \\
\hline \multirow{2}{*}{$22 \mathrm{H}$} & Digital & 10 & .042 & .0155 & .020 & .070 & \multirow{2}{*}{.048} \\
\hline & Conventional & 10 & .074 & .0444 & .009 & .139 & \\
\hline \multirow{2}{*}{$25 \mathrm{H}$} & Digital & 10 & .059 & .0315 & .018 & .113 & \multirow{2}{*}{.024} \\
\hline & Conventional & 10 & .108 & .0537 & .039 & .198 & \\
\hline
\end{tabular}

Table 2: Average site-level inaccuracies from digital and conventional impressions.

\begin{tabular}{|c|c|c|c|c|c|c|}
\hline \multicolumn{2}{|c|}{} & N & Mean & $\begin{array}{c}\text { Standard } \\
\text { Deviation }\end{array}$ & Reference & p-value \\
\hline \multirow{5}{*}{ Digital } & I.12V & 10 & .036 & .0160 & 0 & .000 \\
\cline { 2 - 7 } & $\mathrm{I} .15 \mathrm{~V}$ & 10 & .029 & .0204 & 0 & .001 \\
\cline { 2 - 7 } & $\mathrm{I} .22 \mathrm{~V}$ & 10 & .028 & .0157 & 0 & .000 \\
\cline { 2 - 7 } & $\mathrm{I} .25 \mathrm{~V}$ & 10 & .052 & .0288 & 0 & .000 \\
\cline { 2 - 7 } & $\mathrm{I} .12 \mathrm{H}$ & 10 & .051 & .0299 & 0 & .000 \\
\cline { 2 - 7 } & $\mathrm{I} .15 \mathrm{H}$ & 10 & .027 & .0231 & 0 & .006 \\
\cline { 2 - 7 } & $\mathrm{I} .22 \mathrm{H}$ & 10 & .042 & .0155 & 0 & .000 \\
\cline { 2 - 7 } & $\mathrm{I} .25 \mathrm{H}$ & 10 & .059 & .0315 & 0 & .000 \\
\hline \multirow{5}{*}{ tional } & $\mathrm{I} .12 \mathrm{~V}$ & 10 & .079 & .0454 & 0 & .000 \\
\cline { 2 - 7 } & $\mathrm{I} .15 \mathrm{~V}$ & 10 & .106 & .0502 & 0 & .000 \\
\cline { 2 - 7 } & $\mathrm{I} .22 \mathrm{~V}$ & 10 & .100 & .0897 & 0 & .007 \\
\cline { 2 - 7 } & $\mathrm{I} .25 \mathrm{~V}$ & 10 & .092 & .0513 & 0 & .000 \\
\cline { 2 - 7 } & $\mathrm{I} .12 \mathrm{H}$ & 10 & .085 & .0555 & 0 & .001 \\
\cline { 2 - 7 } & $\mathrm{I} .15 \mathrm{H}$ & 10 & .105 & .0740 & 0 & .002 \\
\cline { 2 - 7 } & $\mathrm{I} .22 \mathrm{H}$ & 10 & .074 & .0444 & 0 & .001 \\
\cline { 2 - 7 } & $\mathrm{I} .25 \mathrm{H}$ & 10 & .108 & .0537 & 0 & .000 \\
\hline
\end{tabular}

Table 3: Average imprecision of measurements with reference values. from this study suggests that it is technically possible to obtain an All-on-Four impression using the existing technology.

There appears to be scientific evidence in the available literature, regarding the superiority of the digital intraoral scanning method in relation to the conventional, non-splinted elastomeric impression technique using either an open or a closed custom tray for complete-arch impressions [19,20].

In contrast, there is also available evidence supporting the significant statistical superior accuracy of the non-splinted, opentray, conventional elastomeric impression technique over the IOS impression for complete-arch implant rehabilitation [21-23]. This lack of consensus can be attributed to factors such as the study design, the different IOS device software and hardware used.

Ender and Mehl [24] evaluated several intraoral scanners: CEREC Bluecam $^{\circledR}$, CEREC Omnicam $^{\circledR}$, Cadent iTero ${ }^{\circledR}$, and the Lava $\operatorname{COS}^{\circledR}$, on a complete dental arch, stating that these had local deviations and that their accuracy depended largely on the type and 
technique of scanning. The same goes for Mangano., et al. [25], who compared the precision of the following intraoral scanners: $\operatorname{Trios}^{\circledR}$, CS $3500^{\circledR}$, Zfx Intrascan ${ }^{\circledR}$, Planscan ${ }^{\circledR}$, in oral implantology impressions. For Giménez., et al. [26] an implant angulation of even up 45 degrees does not affect the statistically significant accuracy of intraoral scanners in the case of Cadent iTero ${ }^{\circledR}$ and the Lava $\operatorname{COS}^{\circledR}$.

While the digitization of a single implant regardless of its angulation remains predictable, the digitization of a complete edentulous arch with multiple implants leaves much to be desired in the precision of intraoral scanners [27].

Although the in vitro results of the conducted study were very promising, many factors related to the oral cavity can negatively influence the accuracy of digital impressions; such as lack of space, patient and the tongue movement, in addition to the saliva flow [28].

The precision of digital impressions may be related to the delicate clinical procedures required to complete the conventional impressions. Indeed, incorrect connections of the affected components, excessive dimensional changes in the impression-taking materials (plaster, polyether and other), minor movements caused by unscrewing the transfer copings as well as screwing implant analogs should be noted.

The statistical trends in the results for the digital impression group versus the conventional impression group are consistent with many previous studies [29,30]. Ours even favors the use of intraoral scanners at the expense of conventional impressions in view of the orientation of our statistical analysis.

However, this experimentation has several limitations. First, the comparisons between the two digital and conventional impressions groups were based on virtual measurements, while considering that the results of the E3 desktop scanner (3Shape) were immutable. Second, the measurements were made following a reference point in the vertical direction and another one in the horizontal plane which eliminates the three-dimensional character which is to be considered. Finally, the implants were placed close together in the anterior region and were not spread to the posterior region of the maxilla, so the results of the in vitro study must be taken cautiously due to the different environment that exists in the mouth (tongue, cheeks, opening limitation).
Digital implant dentistry is growing in popularity and has good potential. However, further studies are needed to assess and compare the clinical accuracy of digital impression techniques versus conventional ones in completely edentulous patients with significantly more than four implants [31].

In addition, the entire digital workflow, from planning to final rehabilitation, must be evaluated and compared to conventional methods in terms of saving time, learning curves, accuracy and cost savings.

Digital impressions not only reduce patient discomfort and labor time dramatically, but also simplify clinical procedures, eliminating plaster models and allowing better communication with the dental technician and patients [32]. Likewise, digital impressions aim to speed up and systematize production lines as well as digitally control them in order to reduce inaccuracies. Indeed, reducing the number of steps and simplifying the chains reduce the loss of information and the potential sources of error [33].

Current intraoral scanners are precise enough to perform several implant-retained prosthetic restorations for edentulous patients.

\section{Conclusion}

Considering the limitations of the present in vitro study, the following conclusions can be drawn: for an All-on-Four with both anterior parallel implants and two others inclined at 45 degrees distally at the level of the second premolars, digital impression deviations were smaller than those associated with conventional techniques.

As a result, digital implants impressions on an edentulous model were able to meet and exceed the accuracy of conventional implants impressions. However, further in vivo studies are needed to confirm the results obtained.

\section{Source of Support}

The authors would like to thank PRODENT s.a.l. Lebanon, Mr. Paul Daniel for supplying the implants impressions accessories, as well as Mr. Elie Choueiry for his work on the superimposition software.

Citation: Elie Nasr., et al. "Accuracy of Digital Vs Conventional Implant Impressions for an All-on-Four Treatment Concept". Acta Scientific Dental Sciences 5.11 (2021): 98-106. 


\section{Conflict of Interest}

None.

\section{Bibliography}

1. Maló P., et al. "The All-on-4 concept for full-arch rehabilitation of the edentulous maxillae: A longitudinal study with 5-13 years of follow-up". Clinical Implant Dentistry and Related Research 21.4 (2019): 538-549.

2. Maló P., et al. "The All-on-4 treatment concept for the rehabilitation of the completely edentulous mandible: A longitudinal study with 10 to 18 years of follow-up". Clinical Implant Dentistry and Related Research 21.4 (2019): 565-577.

3. Attard NJ., et al. "Long-term treatment costs associated with implant-supported mandibular prostheses in edentulous patients". The International Journal of Prosthodontics 18.2 (2005): 117-123.

4. Ting-Shu S and Jian S. "Intraoral Digital Impression Technique: A Review”. The Journal of Prosthodontics 24.4 (2015): 313-321.

5. Soto-Penaloza D., et al. "The all-on-four treatment concept: Systematic review". Journal of Clinical and Experimental Dentistry 9.3 (2017): 474-488.

6. Vasilakos G., et al. "Assessment of different techniques for 3D superimposition of serial digital maxillary dental casts on palatal structures". Scientific Reports 7.1 (2017): 5838.

7. Park T-J., et al. "A method for mandibular dental arch superimposition using 3D cone beam CT and orthodontic 3D digital model". Korean Journal of Orthodontics 42.4 (2012): 169-181.

8. Wesemann C., et al. "Accuracy and efficiency of full-arch digitalization and 3D printing: A comparison between desktop model scanners, an intraoral scanner, a CBCT model scan, and stereolithographic 3D printing". Quintessence International 48.1 (2017): 41-50.

9. Suttin ZB., et al. "System and method for improved intra-oral scanning protocol and calibration. U.S. Patent Application 16.1 (2019): 287-439.
10. Aragón MLC., et al. "Validity and reliability of intraoral scanners compared to conventional gypsum models measurements: a systematic review". European Journal of Orthodontics 38.4 (2016): 429-434.

11. Abrol S., et al. "A Comparative Analysis of Master Casts Obtained using Different Surface Treatments on Impression Copings for Single Tooth Implant Replacement -An In vitro Study". Journal of Clinical and Diagnostic Research 11.8 (2017): 102105.

12. Siadat H., et al. "Comparison of Different Impression Techniques When Using the All-on-Four Implant Treatment Protocol". The International Journal of Prosthodontics 29.3 (2016): 265-270.

13. Gherlone E., et al. "Conventional Versus Digital Impressions for "All-on-Four" Restorations". The International Journal of Oral and Maxillofacial Implants 31.2 (2016): 324-330.

14. Lee S-J and Cho S-B. "Accuracy of five implant impression technique: effect of splinting materials and methods". The Journal of Advanced Prosthodontics 3.4 (2011): 177-185.

15. Hoods-Moonsammy V., et al. "A Comparison of the Accuracy of Polyether, Polyvinyl Siloxane, and Plaster Impressions for Long-Span Implant-Supported Prostheses". The International Journal of Prosthodontics 27.5 (2014): 433-438.

16. Chia VA., et al. "In Vitro Three-Dimensional Accuracy of Digital Implant Impressions: The Effect of Implant Angulation". The International Journal of Oral and Maxillofacial Implants 32.2 (2017): 313-321.

17. Güth J-F., et al. "A new method for the evaluation of the accuracy of full-arch digital impressions in vitro". Clinical Oral Investigations 20.7 (2016): 1487-1494.

18. Zimmermann M., et al. "Intraoral scanning systems-a current overview". International Journal of Computerized Dentistry 18.2 (2015): 101-129.

19. Alikhasi M., et al. "Three-dimensional accuracy of digital impression versus conventional method: effect of implant angulation and connection type". International Journal of Dentistry 4 (2018): 3761750. 
20. Rech-Ortega C., et al. "Comparative in vitro study of the accuracy of impression techniques for dental implants: direct technique with an elastomeric impression material versus intraoral scanner". Medicina Oral, Patologia Oral, Cirugia Bucal 24.1 (2019): 89-95.

21. Kim KR., et al. "Conventional open-tray impression versus intraoral digital scan for implant-level complete-arch impression". Journal of Prosthetic Dentistry 122.6 (2019): 543-549.

22. Tan MY., et al. "Comparison of three-dimensional accuracy of digital and conventional implant impressions: effect of interimplant distance in an edentulous arch". The International Journal of Oral and Maxillofacial Implants 34.2 (2019): 366380.

23. Huang R., et al. "Improved scanning accuracy with newly designed scan bodies: an in vitro study comparing digital versus conventional impression techniques for complete-arch implant rehabilitation". Clinical Oral Implants Research 31.7 (2020): 625-633.

24. Ender A and Mehl A. "In-vitro evaluation of the accuracy of conventional and digital methods of obtaining full-arch dental impressions". Quintessence International 46.1 (2015): 9-17.

25. Mangano FG., et al. "Trueness and Precision of Four Intraoral Scanners in Oral Implantology: A Comparative in Vitro Study". PLoS One 11.9 (2016): 107-163.

26. Giménez B., et al. "Accuracy of a digital impression system based on parallel confocal laser technology for implants with consideration of operator experience and implant angulation and depth". The International Journal of Oral and Maxillofacial Implants 29.4 (2014): 853-862.

27. Chew AA., et al. "Three-Dimensional Accuracy of Digital Implant Impressions: Effects of Different Scanners and Implant Level". The International Journal of Oral and Maxillofacial Implants 32.1 (2017): 70-80.

28. Jivanescu A., et al. "Clinical Factors Influence the Trueness of Intra-oral Scanning". European Journal of Prosthodontics and Restorative Dentistry 27.1 (2019): 51-55.
29. Abizadeh N., et al. "Digital versus plaster study models: how accurate and reproducible are they?" Journal of Orthodontics 39.3 (2012): 151-159.

30. Ribeiro P., et al. "Accuracy of Implant Casts Generated with Conventional and Digital Impressions-An In Vitro Study". International Journal of Environmental Research and Public Health 15.8 (2018): 1599.

31. Kim M-K., et al. "The effect of scanning distance on the accuracy of intra-oral scanners used in dentistry". Clinical Anatomy 32.3 (2019): 430-438.

32. Chochlidakis KM., et al. "Digital versus conventional impressions for fixed prosthodontics: A systematic review and metaanalysis". Journal of Prosthetic Dentistry 116.2 (2016): 184190.

33. Ahlholm P., et al. "Digital Versus Conventional Impressions in Fixed Prosthodontics: A Review: Digital vs. Conventional Impressions in Fixed Prosthodontics". Journal of Prosthodontic 27.1 (2018): 35-41.

Volume 5 Issue 11 November 2021 (C) All rights are reserved by Elie Nasr., et al. 